\title{
Polymer SU-8-based microprobes for neural recording and drug delivery
}

\author{
Ane Altuna ${ }^{1 *}$, Javier Berganzo ${ }^{2}$ and Luis J. Fernández ${ }^{3,4}$ \\ ${ }^{1}$ microLIQUID, Mondragon, Spain, ${ }^{2}$ Microsystems Department, IK4-IKERLAN, Mondragon, Spain, ${ }^{3}$ Centro de Investigación \\ Biomédica en Red, Biomateriales y Nanomedicina (CIBER-BBN), Zaragoza, Spain, ${ }^{4}$ Group of Structural Mechanics and \\ Materials Modelling (GEMM), Aragón Institute of Engineering Research (I3A), Universidad de Zaragoza, Zaragoza, Spain
}

\section{OPEN ACCESS}

Edited by:

Daniel J. Solis,

BioNano Genomics, USA

Reviewed by:

Jose L. Garcia-Cordero,

Center for Research and

Advanced Studies of the National

Polytechnic Institute, Mexico

Jungkyu Jay Kim,

Texas Tech University, USA

*Correspondence:

Ane Altuna,

Goiru 9, 20500, Arrasate, Gipuzkoa,

Spain

anealtuna@microliquid.com

Specialty section:

This article was submitted to

Translational Materials Science,

a section of the journal

Frontiers in Materials

Received: 27 May 2014

Accepted: 30 May 2015

Published: 18 June 2015

Citation:

Altuna A, Berganzo J and

Fernández LJ (2015) Polymer

SU-8-based microprobes for neural recording and drug delivery.

Front. Mater. 2:47.

doi: 10.3389/fmats.2015.00047
This manuscript makes a reflection about SU-8-based microprobes for neural activity recording and drug delivery. By taking advantage of improvements in microfabrication technologies and using polymer SU-8 as the only structural material, we developed several microprobe prototypes aimed to (a) minimize injury in neural tissue, (b) obtain high-quality electrical signals, and (c) deliver drugs at a micrometer precision scale. Dedicated packaging tools have been developed in parallel to fulfill requirements concerning electric and fluidic connections, size and handling. After these advances have been experimentally proven in brain using in vivo preparation, the technological concepts developed during consecutive prototypes are discussed in-depth now.

Keywords: SU-8 probes, drug delivery, neural activity recording, probe packaging, microfabrication techniques

\section{Introduction}

Microprobe development for neuronal applications has been refined to reach minimal invasiveness, high reproducibility, and monolithic integration with microelectronics. Reducing the invasive nature of neural probes is a major step while aiming to interfere with neural activity patterns at the cellular level. To this purpose, advances in microfabrication technology have been exploited to integrate high-density sensing sites into a single miniaturized probe for simultaneous discrimination of large neuronal ensembles. Hence, sophisticated fabrication methods have been developed in the microelectronics industry for over half a century. As a result, high reliability and high-volume production levels have been achieved. Furthermore, improvements in communication between microtechnologists and the neuroscientific community were updated in relation with the growth of connection sites, reduction of external signal influence, and autonomy of the device.

In addition to improvements of electronics for neural applications, microfluidic integration gained interest over the last years. In fact, precise and spatiotemporal controlled delivery by microprobes is considered to be one of the most promising directions for delivering pharmacological compounds directly into specific brain regions. From its beginning, silicon technology pioneered the integration of fluidic microchannels into a probe (Chen et al., 1997). Anisotropic silicon etching, boron etch stop, and thermal oxidation/low-pressure chemical vapor deposition sealing were key steps in putting together microelectrodes and fluidic channels for a first time. Changes in the response properties of isolated neurons during drug delivery confirmed the validity of this approach. Since then, efforts have been devised toward precisely control and monitor fluid movements (Lin and Pisano, 1999; McAllister et al., 2000; Rathnasingham et al., 2004; Neeves et al., 2006; Papageorgiou et al., 2006; Foley et al., 2009; Rohatgi et al., 2009; Pongrácz et al., 2013; Lee et al., 2015). Recently, multi-shank fluidic silicon probes have been developed for neuroscientific applications (Seidl et al., 2010; Frey et al., 2011; John et al., 2011; 
Spieth et al., 2011). Fluidic channels have been successfully integrated into the silicon $3 \mathrm{D}$ configuration via isotropic etching and parylene resealing process.

In parallel, polymer-based microfabrication technology has been developed to deal with invasiveness and brittleness associated with silicon (Subbaroyan et al., 2005; Cheung and Renaud, 2006; Mercanzini et al., 2007, 2008). Parylene technology has already been reported as an option for fluidic applications (Takeuchi et al., 2005). A sacrificial photoresist was defined at specific thickness between two parylene layers and later dissolved to create the cavity. As an alternative to this method, thermal bonding was used (Ziegler et al., 2006). Its main advantages were simplicity, fast production, and the possibility to increase adhesion between the structural layers. Microprobes based on polyimide technology were also developed (Metz et al., 2004). Sputtering and dry etching technique were used to integrate electrodes on the polymer, and lamination technique was implemented to create the channel. It was concluded that this technology offers versatibility for the design of electrodes and channels in terms of size, number, and position. Also, benzocyclobutene (BCB) has been combined with silicon technology to include both, electrodes and fluidics (Lee et al., 2004).

Our group has already exploited polymer SU-8 microfabrication technology in order to integrate multiple electrodes and fluidic channels into a single probe. Over the last years, it has been demonstrated that SU-8 polymer provides optimal properties for neural applications (Lu et al., 2006; Cho et al., 2008; Fernández et al., 2009; Altuna et al., 2010, 2012, 2013). Its high-aspect ratio capability has enabled good dimensional control, and therefore, probes with vertical sidewalls provided a clean insertion into the soft tissue. In addition, high uniformity and adhesion properties ensured the positioning of the metallic tracks on top of the polymer. Recently, planar electrodes were integrated at probe surface and signal recording improved significantly (Altuna et al., 2012). Bonding at low temperature and pressure was first suggested as an optimal technique to create embedded microchannels (Blanco et al., 2004; Agirregabiria et al., 2005; Arroyo et al., 2007; Fernández et al., 2009). Now, even lower temperature combined with higher pressure has been used to avoid internal forces and to guarantee probe planarity. In all cases, the mechanical suitability of SU-8-based probes were experimentally tested for neural application ( $\mathrm{Lu}$ et al., 2006; Cho et al., 2008; Fernández et al., 2009; Altuna et al., 2010, 2012, 2013). In addition, new achievements regarding probe packaging are addressed in this document. Packaging is known to be a major cost factor in the field of medical microdevices. Therefore, simple, robust, and re-usable housing becomes highly desirable, especially when life-limiting components are used. When electric and fluidic functions are integrated into a single microdevice, all connections have to fit into tight space restriction. Accordingly, our microfluidic SU-8 probes have been displayed every time by a dedicated packaging based on high-resolution 3D printing techniques (Fernández et al., 2009; Altuna et al., 2010, 2012, 2013).

\section{Viability Study of SU-8 Microprobes for Neural Applications}

Our first SU-8 prototype included novel design and material concepts to allow a flexible microprobe fabrication for a wide range of neural applications (Figure 1A). Polymer SU-8 was chosen as an exclusive structural material aimed to integrate miniaturized electrodes and fluidic microchannels. Insertion area was chosen to be considerably larger than typical silicon-based probes during the first stage of development $(400 \mu \mathrm{m} \times 220 \mu \mathrm{m})$ to ensure mechanical stiffness of the SU-8 shank. Six electrodes with a size of $50 \mu \mathrm{m} \times 50 \mu \mathrm{m}$ were placed close to the tip to facilitate recording of neuronal signals close to the delivery radius. Photolithography technique was used to define the pattern of the probe, and metallic layers were coated on top of a thick undeveloped SU-8 layer for the first time by a controlled sputtering process. In addition, SU-8 made microchannels were processed on top by thermocompression based on a recipe developed by Ikerlan/IK4 (Blanco et al., 2004; Agirregabiria et al., 2005; Arroyo et al., 2007). Preliminary electrical characterization verified the sensing capability of the probe and biological tests confirmed clean delivery into the brain (Fernández et al., 2009).

\section{Microprobe Development: Minimal Tissue Damage and Improved Electrical Recording}

Once functional viability of the first prototype was verified, the dimensions of the insertion area were reduced in an order of magnitude to limit injury of the neural tissue. Probe lengths and designs were adapted for in vitro experiments and a tetrode configuration was implemented at the tip in order to record activity from small neuronal groups and to discriminate independent neurons (Figure 1B). The electrode diameter was set at $20 \mu \mathrm{m}$, quite comparable to neuron size. In relation with the fabrication procedure, baking conditions and photolithography exposure dose were optimized to gain resolution of such reduced patterns. This design enabled the recording of extracellular action potentials with peak-to-peak amplitudes comparable to conventional tetrode (Altuna et al., 2010). Then, in an effort to improve the recording capability of the tetrode, a revolutionary fabrication sequence was developed (Altuna et al., 2012). The typical gap between the sensing site and tissue was fully eliminated and planar electrodes were located at the probe surface level (Figure 1C). In addition, the thickness of the nearest SU-8 layer was reduced to minimize injury at depth brain in vivo recordings. This advances positively impacted on recording capability, as tested experimentally in the rat hippocampus in vivo. Action potentials emerging from individual neurons with high peak-to-peak amplitudes were measured using SU-8 probes.

At this stage, a novel fabrication procedure was developed to better integrate planar electrodes and well-defined microchannels in reduced probe sizes. Once the technological scope was demonstrated (Figure 1D), we moved forward in order to increase specificity in the application field. The position of the outlets was 


\section{A}

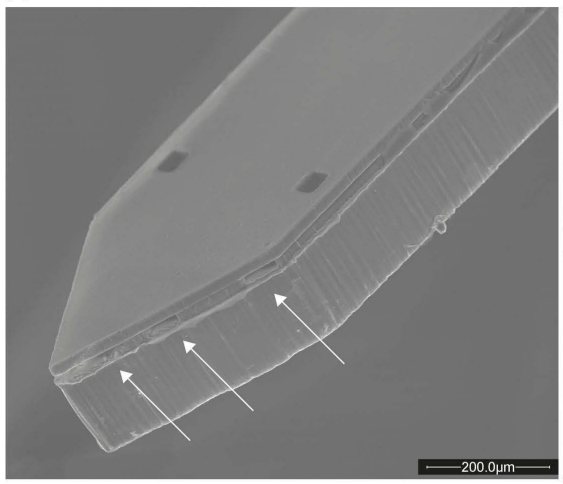

B
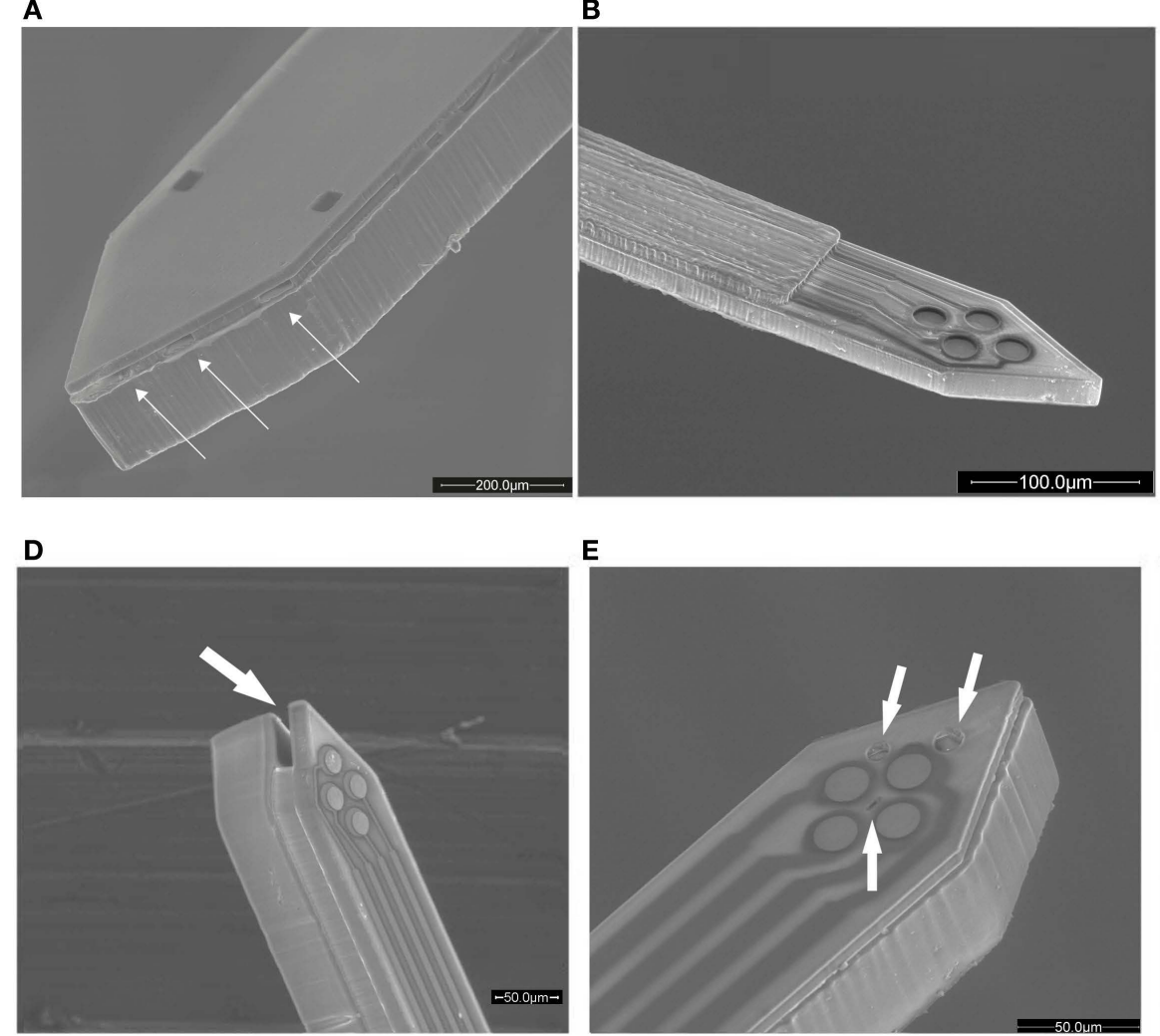

E

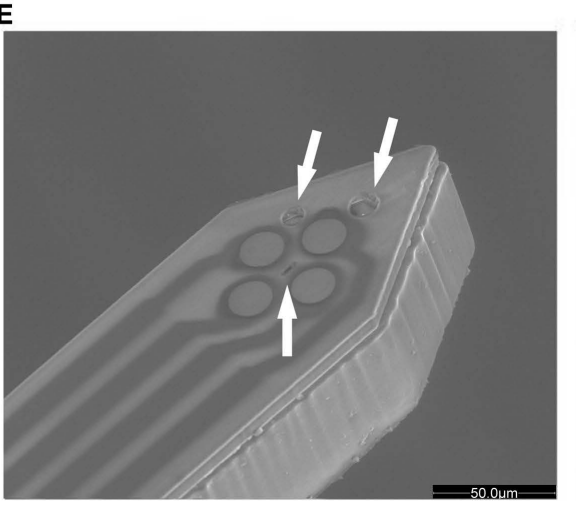

C

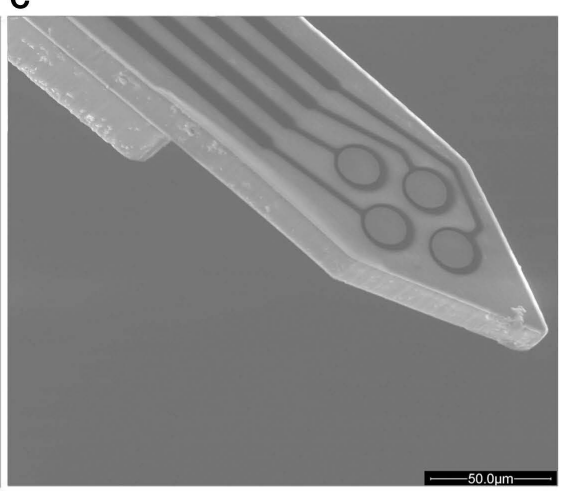

$\mathbf{F}$

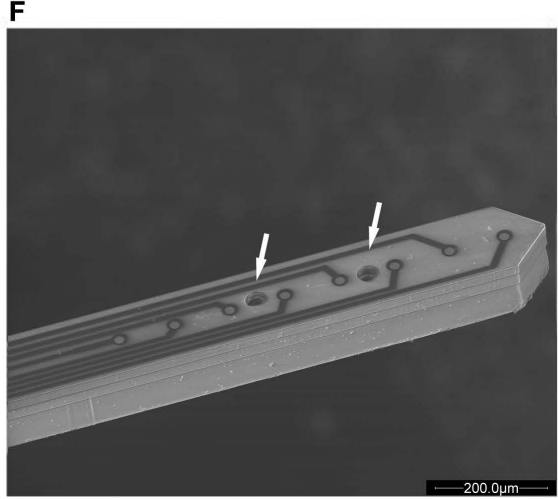

FIGURE 1 | Evolution of the SU-8 microprobe shown by scanning electron microscopy (SEM) figures: (A) initial electrode configuration and a fluidic channel with multiple lateral outlets, (B) first tetrode version for in vitro applications, (C) the tetrode at the probe surface level, (D) fluidic implementation to the tetrode version, (E) the tetrode with three fluidic outlets in the same face of the probe, $(\mathbf{F})$ eight electrodes in a row and two fluidic outlets in the same face of the probe. The outlets are indicated with arrows. changed from the lateral side of the probe to the top side in order to place the outlets in the same level as the electrodes (Figure 1E). Additionally, a linear electrode configuration was developed to facilitate recording from several brain layers simultaneously. In relation to fluidics, two channels with internal dimensions of $40 \mu \mathrm{m} \times 20 \mu \mathrm{m}$ were added. Both channels had independent inlet and outlet ports in order to control drug delivery individually (Figure 1F).

\section{Development of Packaging Tools}

In parallel to the progress of probe design and microfabrication, packaging was adjusted to each probe prototype. The first packaging was aimed to integrate multiple sensing sites and a single fluidic connection (Fernández et al., 2009). A proper fixation of the probe into the capsule and an improved sealing ensured pressure application up to 4 bar (Figure 2A). Next probe design required exclusively electric connection and to facilitate handling in typical experimental setups available at neuroscience research laboratories, therefore, a printed circuit board (PCB) with 16 electric contacts was developed (Altuna et al., 2010). A conductive adhesive between the microelectrode and electric pad of the PCB enabled a robust connection for electric data transfer (Figure 2B). The third design added complexity due to its eight electric and fluidic ports in such a reduced device (Figure 2C). With this prototype we recorded for the first time neural ensemble activity in response to local drug delivery at a microscale resolution (Altuna et al., 2013).

Specific details of early SU-8 microprobe prototypes (Figures 1A-D) and their corresponding packaging (Figures 2A-C) have been already published. Since the last probe and packaging prototype provided us reliable experimental results, both probe and packaging designs are now under a commercialization program by microLIQUID (www.microliquid.com).

\section{Discussion and Future}

This manuscript summarizes the evolution of our SU-8 microprobe's design and packaging since its beginning up to the present. The first probe prototype was the master key, since it was demonstrated the viability of polymer SU-8 as structural material for neural applications at fine scale. In each consecutive prototype, electrode design was improved for better signal-to-noise ratio and the fluidic configuration evolved to multiple independent 

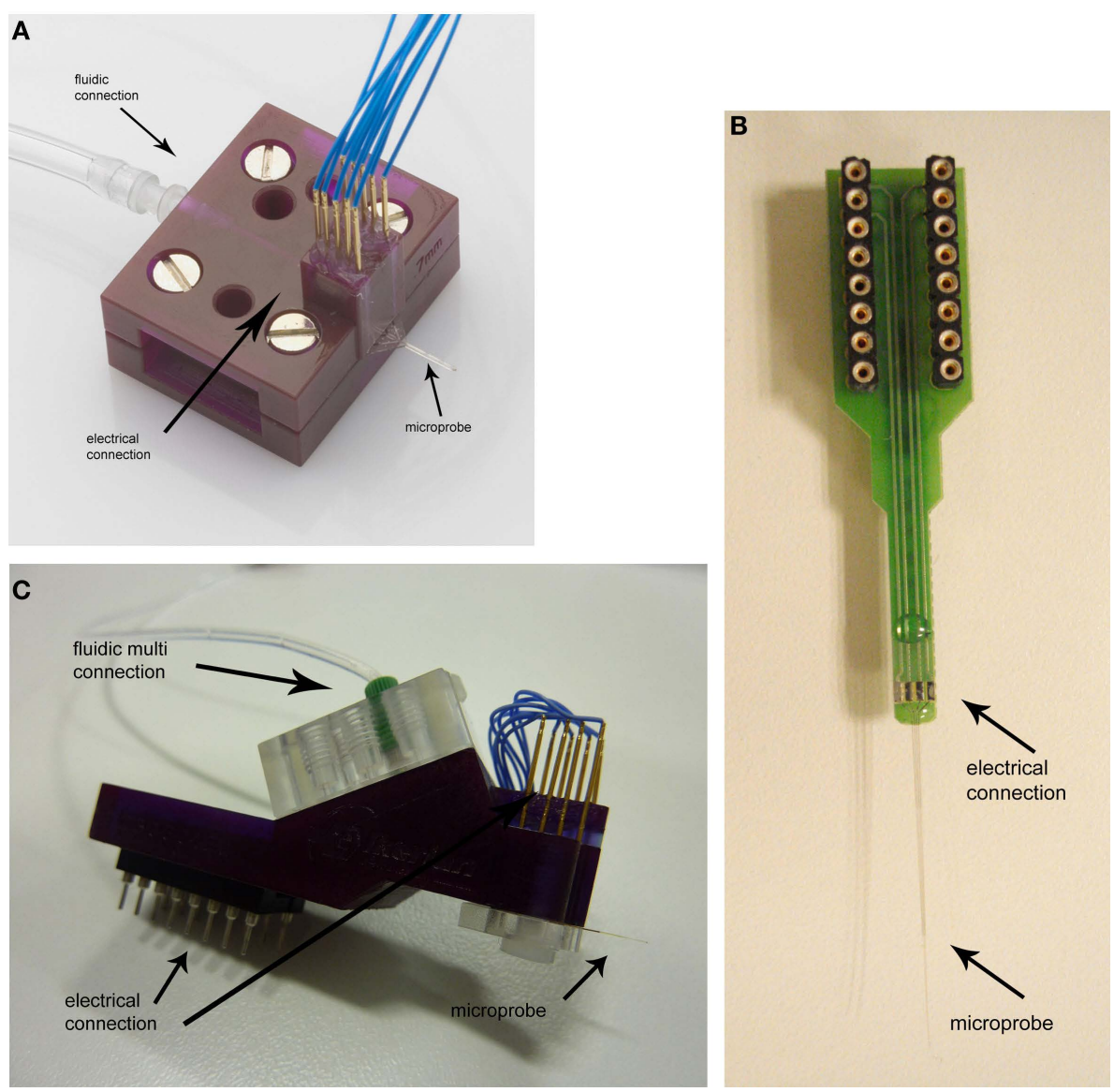

FIGURE 2 | SU-8 probe-packaging designs: (A) first packaging design with eight electric contacts and a fluidic connection, (B) PCB with tetrode connections, and (C) novel packaging with multiple electric and fluidic connections.

microchannels facing the same side of the electrode. Accordingly, our three packaging designs not only conceived to ensure reliable connections but also easy manipulation. Just as technological aspects of the probe and packaging were improved over time, the complexity of the experiments also increased until simultaneous functionality for depth electrical recording and drug delivery in the brain at a scale of few hundreds of micrometers was demonstrated. Future steps will be focused on chronic neural applications aimed to gain better spatiotemporal control of drug delivery and

\section{References}

Agirregabiria, M., Blanco, F. J., Berganzo, J., Arroyo, M. T., Fullaondo, A., Mayora, K., et al. (2005). Fabrication of SU-8 multilayer microstructures based on successive CMOS compatible adhesive bonding and releasing steps. Lab. Chip 5, 545-552. doi:10.1039/b500519a

Altuna, A., Bellistri, E., Cid, E., Aivar, P., Gal, B., Berganzo, J., et al. (2013). SU-8 based microprobes for simultaneous neural depth recording and drug delivery in the brain. Lab. Chip 13, 1422-1430. doi:10.1039/c3lc41364k

Altuna, A., Gabriel, G., De La Prida, L. M., Tijero, M., Guimerá, A., Berganzo, J., et al. (2010). SU-8-based microneedles for in vitro neural applications. J. Micromech. Microeng. 20. doi:10.1088/0960-1317/20/6/064014 recording during long-term experiments. In this direction, specific packaging tools will be manufactured with the aim to get a compact probe-packaging ensemble.

\section{Acknowledgments}

The authors would like to thank the Biomedical Application Group at CNM, Barcelona and the work team of Liset Menendez de la Prida at Instituto Cajal, Madrid.

Altuna, A., Menendez de la Prida, L., Bellistri, E., Gabriel, G., Guimerá, A., Berganzo, J., et al. (2012). SU-8 based microprobes with integrated planar electrodes for enhanced neural depth recording. Biosens. Bioelectron. 37, 1-5. doi:10.1016/j. bios.2012.03.039

Arroyo, M. T., Fernández, L. J., Agirregabiria, M., Ibãez, N., Aurrekoetxea, J., and Blanco, F. J. (2007). Novel all-polymer microfluidic devices monolithically integrated within metallic electrodes for SDS-CGE of proteins. J. Micromech. Microeng. 17, 1289-1298. doi:10.1088/0960-1317/17/7/011

Blanco, F. J., Agirregabiria, M., Garcia, J., Berganzo, J., Tijero, M., Arroyo, M. T., et al. (2004). Novel three-dimensional embedded SU-8 microchannels fabricated using a low temperature full wafer adhesive bonding. J. Micromech. Microeng. 14, 1047-1056. doi:10.1088/0960-1317/14/7/027 
Chen, J., Wise, K. D., Hetke, J. F., and Bledsoe, S. C. Jr. (1997). A multichannel neural probe for selective chemical delivery at the cellular level. IEEE Trans. Biomed. Eng. 44, 760-769. doi:10.1109/10.605435

Cheung, K. C., and Renaud, P. (2006). BioMEMS for medicine: on-chip cell characterization and implantable microelectrodes. Solid State Electron. 50, 551-557. doi:10.1016/j.sse.2006.03.023

Cho, S. H., Lu, H. M., Cauller, L., Romero-Ortega, M. I., Lee, J. B., and Hughes, G. A. (2008). Biocompatible SU-8-based microprobes for recording neural spike signals from regenerated peripheral nerve fibers. IEEE Sens. J. 8, 1830-1836. doi:10.1109/ JSEN.2008.2006261

Fernández, L. J., Altuna, A., Tijero, M., Gabriel, G., Villa, R., Rodríguez, M. J., et al. (2009). Study of functional viability of SU-8-based microneedles for neural applications. J. Micromech. Microeng. 19. doi:10.1088/0960-1317/19/2/025007

Foley, C. P., Nishimura, N., Neeves, K. B., Schaffer, C. B., and Olbricht, W. L. (2009). Flexible microfluidic devices supported by biodegradable insertion scaffolds for convection-enhanced neural drug delivery. Biomed. Microdevices 11, 915-924. doi:10.1007/s10544-009-9308-6

Frey, O., Holtzman, T., McNamara, R. M., Theobald, D. E. H., Van Der Wal, P. D., De Rooij, N. F., et al. (2011). Simultaneous neurochemical stimulation and recording using an assembly of biosensor silicon microprobes and SU-8 microinjectors. Sens. Actuators B Chem. 154, 96-105. doi:10.1016/j.snb.2010.01.034

John, J., Li, Y., Zhang, J., Loeb, J. A., and Xu, Y. (2011). Microfabrication of 3D neural probes with combined electrical and chemical interfaces. J. Micromech. Microeng. 21. doi:10.1088/0960-1317/21/10/105011

Lee, H. J., Son, Y., Kim, J., Lee, C. J., Yoon, E. S., and Cho, I. J. (2015). A multichannel neural probe with embedded microfluidic channels for simultaneous in vivo neural recording and drug delivery. Lab. Chip 15, 1590-1597. doi:10.1039/c4lc01321b

Lee, K., He, J., Clement, R., Massia, S., and Kim, B. (2004). Biocompatible benzocyclobutene (BCB)-based neural implants with micro-fluidic channel. Biosens. Bioelectron. 20, 404-407. doi:10.1016/j.bios.2004.02.005

Lin, L., and Pisano, A. P. (1999). Silicon-processed microneedles. J. Microelectromech. Syst. 8, 78-84. doi:10.1109/84.749406

Lu, H., Cho, S.-H., Lee, J.-B., Romero-Ortega, M., Cauller, L., and Hughes, G. (2006). "SU8-based micro neural probe for enhanced chronic in-vivo recording of spike signals from regenerated axons," in Proceedings of IEEE Sensors, Art. No. 4178557, 66-69.

McAllister, D. V., Allen, M. G., and Prausnitz, M. R. (2000). Microfabricated microneedles for gene and drug delivery. Annu Rev Biomed Eng 2, 289-313.

Mercanzini, A., Cheung, K., Buhl, D. L., Boers, M., Maillard, A., Colin, P., et al. (2008). Demonstration of cortical recording using novel flexible polymer neural probes. Sens. Actuators A Phys. 143, 90-96. doi:10.1016/j.sna.2007.07.027

Metz, S., Bertsch, A., Bertrand, D., and Renaud, P. (2004). Flexible polyimide probes with microelectrodes and embedded microfluidic channels for simultaneous drug delivery and multi-channel monitoring of bioelectric activity. Biosens. Bioelectron. 19, 1309-1318. doi:10.1016/j.bios.2003.11.021

Neeves, K. B., Lo, C. T., Foley, C. P., Saltzman, W. M., and Olbricht, W. L. (2006). Fabrication and characterization of microfluidic probes for convection enhanced drug delivery. J. Control. Release 111, 252-262. doi:10.1016/j.jconrel.2005.11.018

Papageorgiou, D. P., Shore, S. E., Bledsoe, S. C. Jr., and Wise, K. D. (2006). A shuttered neural probe with on-chip flowmeters for chronic in vivo drug delivery. J. Microelectromech. Syst. 15, 1025-1033. doi:10.1109/JMEMS.2005.863733

Pongrácz, A., Fekete, Z., Márton, G., Bérces, Z., Ulbert, I., and Fürjes, P. (2013). Deepbrain silicon multielectrodes for simultaneous in vivo neural recording and drug delivery. Sens. Actuators B Chem. 189, 97-105. doi:10.1039/c4lc01321b

Rathnasingham, R., Kipke, D. R., Bledsoe, S. C. Jr., and McLaren, J. D. (2004). Characterization of implantable microfabricated fluid delivery devices. IEEE Trans. Biomed. Eng. 51, 138-145. doi:10.1109/TBME.2003.820311

Rohatgi, P., Langhals, N. B., Kipke, D. R., and Patil, P. G. (2009). In vivo performance of a microelectrode neural probe with integrated drug delivery laboratory investigation. Neurosurg. Focus 27. doi:10.3171/2009.4.FOCUS0983

Seidl, K., Spieth, S., Herwik, S., Steigert, J., Zengerle, R., Paul, O., et al. (2010). In-plane silicon probes for simultaneous neural recording and drug delivery. J. Micromech. Microeng. 20. doi:10.1088/0960-1317/20/10/105006

Spieth, S., Brett, O., Seidl, K., Aarts, A. A. A., Erismis, M. A., Herwik, S., et al. (2011). A floating 3D silicon microprobe array for neural drug delivery compatible with electrical recording. J. Micromech. Microeng. 21. doi:10.1088/0960-1317/21/12/125001

Subbaroyan, J., Martin, D. C., and Kipke, D. R. (2005). A finite-element model of the mechanical effects of implantable microelectrodes in the cerebral cortex. J. Neural Eng. 2, 103-113. doi:10.1088/1741-2560/2/4/006

Takeuchi, S., Ziegler, D., Yoshida, Y., Mabuchi, K., and Suzuki, T. (2005). Parylene flexible neural probes integrated with microfluidic channels. Lab. Chip 5, 519-523. doi:10.1039/b417497f

Ziegler, D., Suzuki, T., and Takeuchi, S. (2006). Fabrication of flexible neural probes with built-in microfluidic channels by thermal bonding of Parylene. J. Microelectromech. Syst. 15, 1477-1482. doi:10.1109/JMEMS.2006.879681

Conflict of Interest Statement: The authors declare that the research was conducted in the absence of any commercial or financial relationships that could be construed as a potential conflict of interest.

Copyright (C) 2015 Altuna, Berganzo and Fernández. This is an open-access article distributed under the terms of the Creative Commons Attribution License (CC BY). The use, distribution or reproduction in other forums is permitted, provided the original author(s) or licensor are credited and that the original publication in this journal is cited, in accordance with accepted academic practice. No use, distribution or reproduction is permitted which does not comply with these terms. 